\title{
One-step synthesis of dandelion-like lanthanum titanate nanostructures for enhanced photocatalytic performance
}

\author{
Zhong Huang ${ }^{1}$, Jianghao Liu', Liang Huang ${ }^{1}$, Liang Tian', Sen Wang ${ }^{1}$, Gen Zhang ${ }^{1}$, Junyi Li ${ }^{1}$, Feng Liang ${ }^{1}$,
} Haijun Zhang (1), Quanli Jia ${ }^{2}$ and Shaowei Zhang ${ }^{3}$

\begin{abstract}
The rational design of nanomaterials with distinct exposed facets is of great importance for improving the physicochemical properties of these materials and for the study of structure-activity relationships. This work describes the first synthesis of lanthanum titanate $\left(\mathrm{La}_{2} \mathrm{Ti}_{2} \mathrm{O}_{7}, \mathrm{LTO}\right)$ with dandelion-like nanostructures via the molten salt method. The lowest synthesis temperature of $700^{\circ} \mathrm{C}$ is at least $200^{\circ} \mathrm{C}$ lower than that required by other methods. The dandelion structure consists of well-crystallized LTO nanorods (NRs) with sizes of less than $100 \mathrm{~nm}$ in the radial direction and 300-500 nm in the axial direction, which is different from the widely accepted two-dimensional form. LaOCI microplates were formed as an intermediate substrate for LTO NR growth outwards to the basal surfaces of the $\mathrm{LaOCl}$ crystallites. DFT calculation results showed that the strong LiCl adsorption on the (100) surface led to distinct growth of the (100) and (020) planes, thus promoting the rod-like growth of LTO along the [010] axis. In addition, the photocatalytic performance of as-prepared LTO was evaluated by determining the degradation of rhodamine B. The results suggested that the as-prepared LTO could markedly enhance the photocatalytic activity as a result of the surface heterojunction of coexposed $\{100\}$ and $\{002\}$ facets in LTO NRs.
\end{abstract}

\section{Introduction}

Lanthanum titanate $\left(\mathrm{La}_{2} \mathrm{Ti}_{2} \mathrm{O}_{7}, \mathrm{LTO}\right)$ with a layered perovskite structure has been extensively investigated in recent decades because of its unique properties, such as ferroelectric properties ${ }^{1,2}$, dielectric properties ${ }^{3}$, and photocatalytic properties $^{4-8}$. In the last case, LTO has been widely reported to exhibit good photocatalytic performance for $\mathrm{CO}_{2}$ conversion ${ }^{4}$, organic pollutant degradation $^{5,6}$, water splitting ${ }^{7,8}$, etc.

LTO has been prepared by hydrothermal synthesis ${ }^{9,10}$, the sol-gel method ${ }^{11}$, coprecipitation synthesis ${ }^{2}$ and the

Correspondence: Liang Huang (huangliang1986@wust.edu.cn) or

Haijun Zhang (zhanghaijun@wust.edu.cn) or Shaowei Zhang

(s.zhang@exeter.ac.uk)

${ }^{1}$ The State Key Laboratory of Refractories and Metallurgy, Wuhan University of Science and Technology, Wuhan 430081, China

${ }^{2}$ Henan Key Laboratory of High Temperature Functional Ceramics, Zhengzhou University, Zhengzhou 450052, China

Full list of author information is available at the end of the article. conventional solid-state method $(\mathrm{CSSM})^{12}$. However, these synthetic processes typically yield low surface areas or require relatively high temperatures $\left(>900^{\circ} \mathrm{C}\right)$. A facile synthetic method, termed molten salt synthesis (MSS), was applied to the synthesis of $\mathrm{LTO}^{7,13-19}$, owing to its advantages of a salt medium to accelerate the homogeneous mixing of reactants and crystal growth at a lower temperature without a temperature gradient. For example, Arney et al. ${ }^{7}$ and Hojamberdiev et al. ${ }^{14,15}$ synthesized plate-like LTO with nanoscale thickness and microscale width at $>1000^{\circ} \mathrm{C}$ by using a single salt or eutectic salts. Cai et al. ${ }^{17-19}$ prepared a step-like LTO nanostructure in $\mathrm{KCl}$ by controlling the heating and cooling rates at $1100^{\circ} \mathrm{C}$. However, in all of the above cases, the as-prepared LTO showed two-dimensional (2D) structures consisting of smooth surfaces ${ }^{7,13-16}$ or step-like surfaces ${ }^{17-19}$. Moreover, the temperatures required $\left(>1000^{\circ} \mathrm{C}\right)$ were almost the same as that used for the CSSM, which is abnormal 
since MSS generally can reduce the lowest synthesis temperature by more than $200^{\circ} \mathrm{C}^{20-24}$. Thus, there is still a significant need to optimize the MSS method to synthesize LTO with an ultrafine size and a diverse structure while markedly reducing the synthesis temperature.

The physicochemical properties of transition metal oxides have been correlated with the different morphologies and specific exposed facets of these materials, especially during photocatalysis. In principle, the exposed facets in a unique shape have distinct band structures and can provide a successive pathway for the migration and separation of photogenerated electrons and holes, thus enhancing the photocatalytic property $^{25-28}$. Therefore, the control of different exposed crystal facets has recently attracted enormous interest. For example, Lin et al. ${ }^{25}$ prepared $\mathrm{WO}_{3}$ nanowires and nanosheets with $\{110\}$ and $\{001\}$, respectively, as the main exposed facets. Because of the distinct charge carrier dynamics of the facets, the photocatalytic activity for benzyl alcohol oxidation of the former was 2.46 times higher than that of the latter. Yu et al. ${ }^{26}$ proposed a new "surface heterojunction" concept to respond to the enhanced photocatalytic performance on $\mathrm{TiO}_{2}$ with coexposed $\{001\}$ and $\{101\}$ facets. The photogenerated electron-hole pairs migrated and separated in these two facets, which can effectively suppress the recombination of the electron-hole pairs. Based on this, Cai et al. ${ }^{17,18}$ reported that the step-like nanostructures of LTO exhibited coexposed $\{010\}$ and $\{012\}$ facets, which formed the surface heterojunction and enhanced the photocatalytic performance. Accordingly, the design and fabrication of LTO with distinct exposed facets is an effective approach to improve the photocatalytic properties and explore the structure-activity relationships of this material.

In response to these findings, a low-temperature MSS route was investigated to tune the distinct exposed facets of LTO; thus, dandelion-like LTO was synthesized with $\mathrm{TiO}_{2}$ and $\mathrm{La}_{2} \mathrm{O}_{3}$ as raw materials and a $\mathrm{LiCl}-\mathrm{KCl}$ binary salt as the molten salt. The synthesis parameters of salt type, reactant/salt ratio and temperature were researched and discussed in detail. The relevant reaction mechanism was also proposed based on the synthesis process and density functional theory (DFT) calculations. To the best of our knowledge, this is the first report on the synthesis of dandelion-like LTO nanostructures. In addition, the photocatalytic activities of rhodamine $\mathrm{B}(\mathrm{RhB})$ degradation over the dandelion-like LTO nanostructure and the widely accepted plate-like nanostructure were also compared. A surface heterojunction formed in the dandelion-like LTO, thus significantly enhancing the photocatalytic activity.

\section{Experimental procedures}

\section{Synthesis of dandelion-like LTO nanostructures}

In a typical synthesis, $\mathrm{TiO}_{2}$ and $\mathrm{La}_{2} \mathrm{O}_{3}$ powders were mixed at a molar ratio of $2: 1$ and then combined with a mass ratio $(1: 1,1: 3,1: 5$ or $1: 7)$ of salts $(\mathrm{KCl}-\mathrm{LiCl}$, $\mathrm{Na}_{2} \mathrm{SO}_{4}-\mathrm{K}_{2} \mathrm{SO}_{4}$ or $\left.\mathrm{NaCl}-\mathrm{KCl}-\mathrm{NaF}\right)$ in the agate mortar. The mixture was transferred to a corundum crucible before being heated at $400-900^{\circ} \mathrm{C}$ for $3 \mathrm{~h}$ in a muffle furnace. Detailed firing conditions and batch compositions of the samples are given in Table S1 in the Supplementary Information. The reacted mass was washed repeatedly with hot deionized water $\left(\sim 50^{\circ} \mathrm{C}\right)$ to remove the salts. Finally, the resultant product powder was ovendried at $80^{\circ} \mathrm{C}$ overnight for further characterization. For comparison, syntheses of LTO with $\mathrm{NaCl}, \mathrm{KCl}$ or $\mathrm{LiCl}$ alone as the molten salt were also carried out according to the previously reported MSS method ${ }^{14,15}$ (Table S1). The obtained products were separated from the remaining salt as described above.

\section{Characterization}

Powder X-ray diffraction (XRD) analysis was carried out on a Philips X'Pert Pro diffractometer (PANalytical, Netherlands). The morphologies of the product powders were examined on a field emission scanning electron microscope (FE-SEM, Nova400NanoSEM, Philips, Netherlands) and a transmission electron microscope (TEM, 2000F, JEOL Ltd., Japan). UV-vis diffuse reflection spectroscopy (DRS) was performed over the range of 200-800 nm on a Shimadzu UV-3600 equipped with an integrating sphere. The Brunauer-Emmett-Teller (BET) surface area was calculated based on the nitrogen adsorption-desorption isotherm (Autosorb-1, Quantachrome, USA). X-ray photoelectron spectroscopy (XPS) was conducted using a VG Multilab 2000 instrument (Thermo Electron Co., USA) equipped with $\mathrm{Al} \mathrm{K}_{\alpha}$ radiation (operated at $300 \mathrm{~W}$ ), and all binding energy positions were calibrated against the $\mathrm{C} 1 \mathrm{~s}$ binding energy at $284.6 \mathrm{eV}$ for adventitious carbon. Electrochemical measurements were conducted on an electrochemical analyzer (CHI660E, Chenhua Instruments, China) with a standard three-electrode system. LTO thin film electrodes were prepared by electrophoretic deposition of LTO powder on fluorine doped tin oxide (FTO) conductive glass supports, as in previous work ${ }^{17}$. The Pt wire acted as the counter electrode, $\mathrm{Ag} / \mathrm{AgCl}$ acted as the reference electrode, and the photoelectrodes acted as the working electrode. A $0.2 \mathrm{M} \quad \mathrm{Na}_{2} \mathrm{SO}_{4}$ aqueous solution was employed as the electrolyte $(\mathrm{pH}=6.8)$. The light source for the photoelectrochemical cell (PEC) was the same as that used for photocatalysis. Electron paramagnetic resonance (EPR) measurements were performed using a Bruker EMX Plus 10/12 spectrometer operating at 
9.41 GHz. Photoluminescence (PL) spectra were obtained on a Shimadzu RF-6000 spectrophotometer with an excitation wavelength of $280 \mathrm{~nm}$.

\section{Photocatalytic measurements}

The photocatalytic performance of the as-prepared LTO was examined in terms of the degradation of $\mathrm{RhB}$ under a $300 \mathrm{~W}$ mercury lamp in a photoreaction apparatus (XPA-4, Xujiang Electromechanical Plant, China). In a typical photocatalytic reaction, $30 \mathrm{mg}$ of the as-prepared LTO was added to $30 \mathrm{~mL}$ of RhB solution $(10 \mathrm{mg} / \mathrm{L})$. In the dark, the suspension was magnetically stirred for $1 \mathrm{~h}$ to reach adsorption-desorption equilibrium. Under UV light irradiation, a $3 \mathrm{~mL}$ aliquot was collected at given time intervals and centrifuged to remove the suspended LTO for measurement. Changes in the RhB concentration were monitored by measuring the absorption intensity at $552 \mathrm{~nm}$ on a UV-vis spectrophotometer (Shimadzu UV3600, Japan) with a quartz cell with an optical pathlength of $10 \mathrm{~mm}$. The photocatalytic degradation reaction kinetics were quantified based on the pseudo-first-order equation:

$$
\ln \left(C / C_{0}\right)=-k t,
$$

where $C_{0}$ is the initial concentration of $\mathrm{RhB}$ solution at $t=0, C$ is the instantaneous $\mathrm{RhB}$ concentration after irradiation for $t$ hours, and $k$ is the apparent kinetic rate constant.

\section{Computational methods}

To understand the growth mechanism and enhanced photocatalytic performance of dandelion-like LTO nanostructures, DFT calculations were conducted under the generalized gradient approximation (GGA) with the Perdew-Burke-Ernzerhof (PBE) exchange-correlation functional as implemented in the VASP $\operatorname{code}^{29-31}$. The monoclinic LTO (space group P21) was chosen as the original crystal structure based on XRD results. The calculated lattice parameters of bulk LTO $(a=7.880, b=$ 5.667, and $c=13.343 \AA$ ) were almost the same as the experimental values $(a=7.800, \quad \mathrm{~b}=5.546$ and $c=$ 13.0112 $\AA$ ). In all calculations and optimization processes, the atoms were allowed to relax. Vacuum slabs of $15 \AA$ were set to avoid interactions between adjacent slabs in the surface model calculations. Brillouin zone integration was performed using a grid spacing of $0.04 \AA^{-1}$ in a Monkhorst-Pack special $k$-point scheme ${ }^{32}$. The interactions between core electrons and ions were described by the projector augmented wave (PAW) method, and the valence electronic states were represented with a planewave basis set with an energy cutoff of $400 \mathrm{eV}$.

The surface energy $(E)$ of LTO (002), (100), and (020) calculated according to the XRD results is defined as follows $^{33-35}$ :

$$
\begin{aligned}
& E=\left(E_{\text {surf }}-n E_{\text {bulk }}\right) / 2 A, \\
& E=\left(E_{\text {surf }+\mathrm{Cl}}-n E_{\text {bulk }}-E_{\mathrm{Cl}}\right) / 2 A,
\end{aligned}
$$

where $E_{\text {surf }}$ is the total energy of the surface, $E_{\mathrm{bulk}}$ is the bulk energy of the unit cell, $E_{\mathrm{Cl}}$ is the energy of the $\mathrm{Cl}$ atom, $E_{\mathrm{surf}+\mathrm{Cl}}$ is the total energy of the Cl-adsorbed surface, $A$ is the surface area, and $n$ represents the number of unit cells that the surface contains.

\section{Results and discussion}

\section{Synthesis and characterization of dandelion-like LTO nanostructures}

First, we investigated the effect of three kinds of representative eutectic salts, i.e., $\mathrm{Na}_{2} \mathrm{SO}_{4}-\mathrm{K}_{2} \mathrm{SO}_{4}$, $\mathrm{NaCl}-\mathrm{KCl}-\mathrm{NaF}$, and $\mathrm{LiCl}-\mathrm{KCl}$, which were frequently used in our previous works ${ }^{20-24}$ for the synthesis of LTO. As shown in Figs. S1 and $\mathrm{S} 2, \mathrm{LiCl}-\mathrm{KCl}$ has the best promoting effect on $\mathrm{LTO}$ synthesis. Thus, $\mathrm{LiCl}-\mathrm{KCl}$ was used as the reaction medium in the following experiments. In addition, the effect of the salt content on LTO synthesis was also discussed. As presented in Figs. S3 and S4, the XRD patterns and corresponding FE-SEM images indicated that (1) no LTO was detected in the absence of $\mathrm{LiCl}-\mathrm{KCl}$, suggesting that the salt is indispensable for LTO synthesis, and (2) a high amount of salt hampered the transformation of the $\mathrm{LaOCl}$ intermediate to LTO. The results revealed that an appropriate content of salt (1:1 or $1: 3$ weight ratio of reactant:salt) is required for LTO formation.

Figure 1 shows the XRD patterns of samples obtained at $400-900{ }^{\circ} \mathrm{C}$ in $\mathrm{LiCl}-\mathrm{KCl}$ with a reactant:salt weight ratio of $1: 3$. When the reaction temperature was $400^{\circ} \mathrm{C}$, no LTO peak was detected in the XRD pattern, and the intermediate-phase $\mathrm{LaOCl}$ was observed as the predominant phase. At $500{ }^{\circ} \mathrm{C}$, LTO was observed, but $\mathrm{LaOCl}$

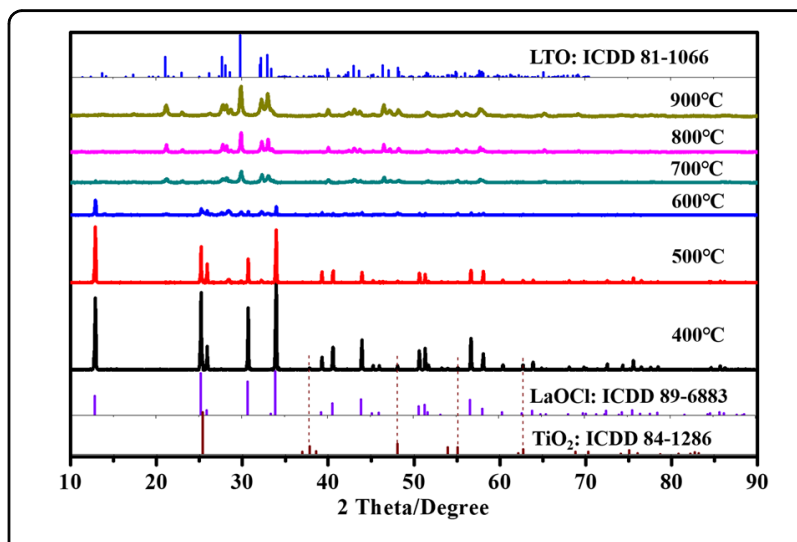

Fig. 1 XRD patterns of samples obtained at various temperatures for $3 \mathrm{~h}$ in $\mathrm{LiCl}-\mathrm{KCl}$ with a reactant:salt weight ratio of 1:3. 

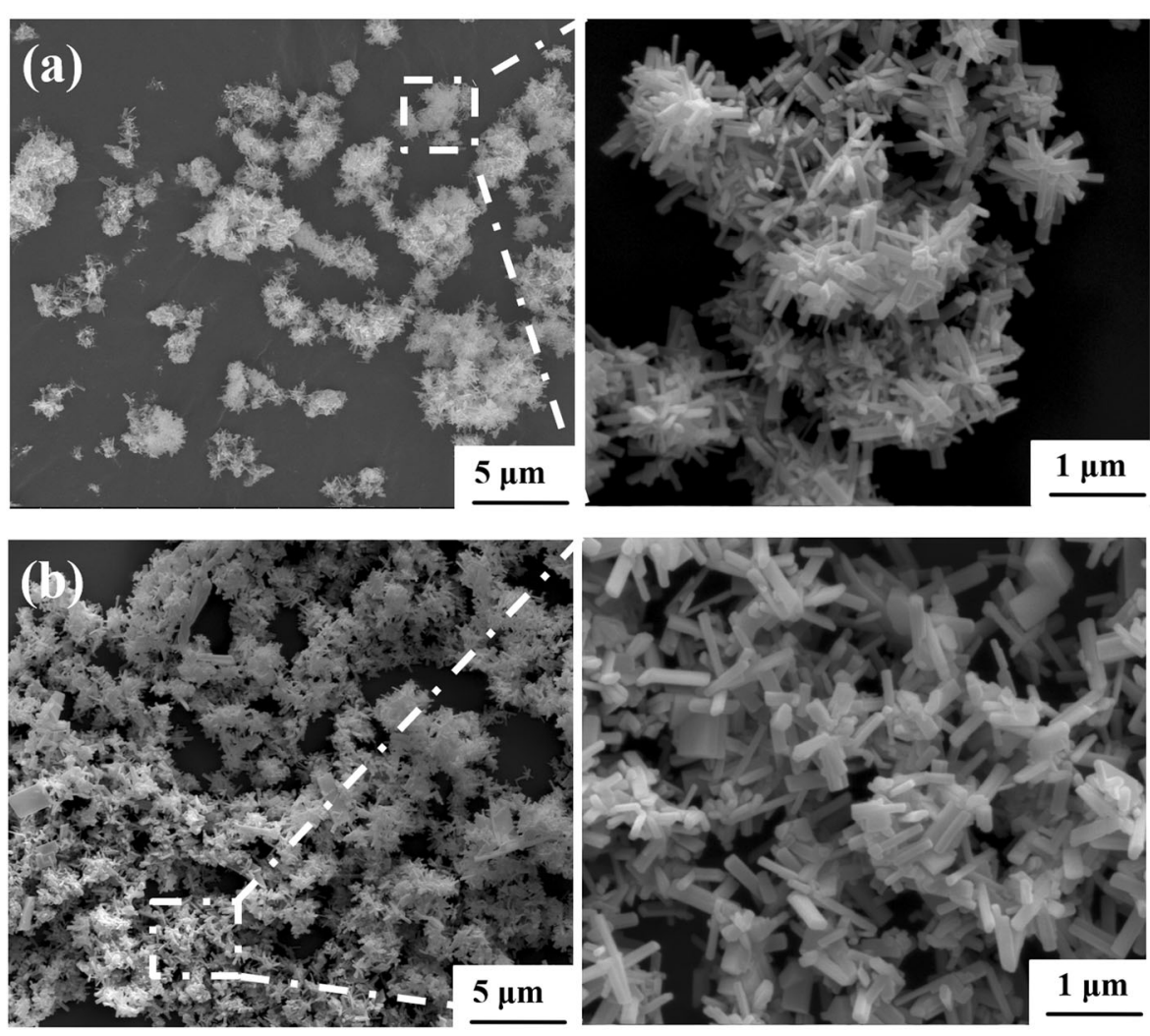

Fig. 2 FE-SEM images of samples obtained at $700^{\circ} \mathrm{C}(\mathbf{a})$ and $900^{\circ} \mathrm{C}$ (b) in LiCl- $\mathrm{KCl}$ with a reactant:salt weight ratio of 1:3.

remained the major phase, suggesting a low degree of LTO formation at this temperature. At $600^{\circ} \mathrm{C}$, although the LTO peak height increased evidently, $\mathrm{LaOCl}$ still remained. Upon increasing the temperature to 700,800 , and $900^{\circ} \mathrm{C}$, only the LTO phase was detected, indicating that the synthesis of LTO had been completed at $\geq 700^{\circ} \mathrm{C}$. The lowest synthesis temperature $\left(700^{\circ} \mathrm{C}\right)$ is much lower than those required by wet-chemical techniques (over $\left.900^{\circ} \mathrm{C}\right)^{2,11}$, CSSM (over $\left.1100^{\circ} \mathrm{C}\right)^{12}$, and MSS (over $\left.1000^{\circ} \mathrm{C}\right)^{7,13-19}$.

Figure S5 and 2 present the FE-SEM images of asprepared samples at different reaction temperatures. At $400^{\circ} \mathrm{C}$, only plate-like $\mathrm{LaOCl}$ with unreacted $\mathrm{TiO}_{2}$ was detected, as shown in Fig. S5a. Upon further increasing the temperature to 500 and $600^{\circ} \mathrm{C}$, in addition to the $\mathrm{LaOCl}$ plates, rod-like LTO with a thickness of tens of nanometers and an orientation outwards to the basal surfaces of the $\mathrm{LaOCl}$ crystallites was clearly observed, as shown in Fig. S5b and c. At $700{ }^{\circ} \mathrm{C}$ (Fig. 2a), the product powders exhibited a dandelion-like structure, consisting of LTO NRs with sizes of less than $100 \mathrm{~nm}$ in the radial direction and $300-500 \mathrm{~nm}$ in the axial direction. By contrast, the sample prepared at $900^{\circ} \mathrm{C}$ (Fig. 2b) showed no observed change in the size of the NRs, while some well-dispersed LTO NRs were formed.
TEM, SAED, and HRTEM were further conducted to characterize the size/morphology and crystalline structure of as-prepared dandelion-like $\mathrm{LTO}$ at $700{ }^{\circ} \mathrm{C}$ in $\mathrm{LiCl}-\mathrm{KCl}$ with a reactant:salt ratio of 1:3. As shown in Fig. 3a and S6, the product exhibited a "blowball" structure (aggregated LTO NRs) with "tufted fruits" that "disperse in the wind" (dispersed LTO NRs with sizes ranging from $278 \mathrm{~nm}$ to $576 \mathrm{~nm}$ in the axial direction), analogous to the structure of the common dandelion. Figure $3 b$ and $d$, as an example, showing the radial morphology of the area indicated by the solid rectangle in Fig. 3a, indicate that the radial direction of the LTO rod exhibited a quasirectangle shape with a length of $65 \mathrm{~nm}$ and a width of $40 \mathrm{~nm}$. The SAED [100] zone axis electron diffraction in Fig. 3c (the selected area in Fig. 3b) indicates that the top and bottom facets of the LTO rod are $\{100\}$. Figure $3 \mathrm{e}$ (indicated by the rectangle in Fig. 3d) and S7 show the HRTEM images for the top facet of LTO NRs, where the two lattice fringes with spacings of 0.509 and $0.227 \mathrm{~nm}$ correspond to the spacing values on the (011) and (020) planes, respectively, of LTO (ICDD 81-1066). These results revealed that the as-formed LTO NRs grew along the [010] axis. Figure $3 \mathrm{f}$ shows the HRTEM images of the front-end facet on the LTO rod (indicated by the circle in Fig. 3d). In addition to the (011) and (020) planes, the 

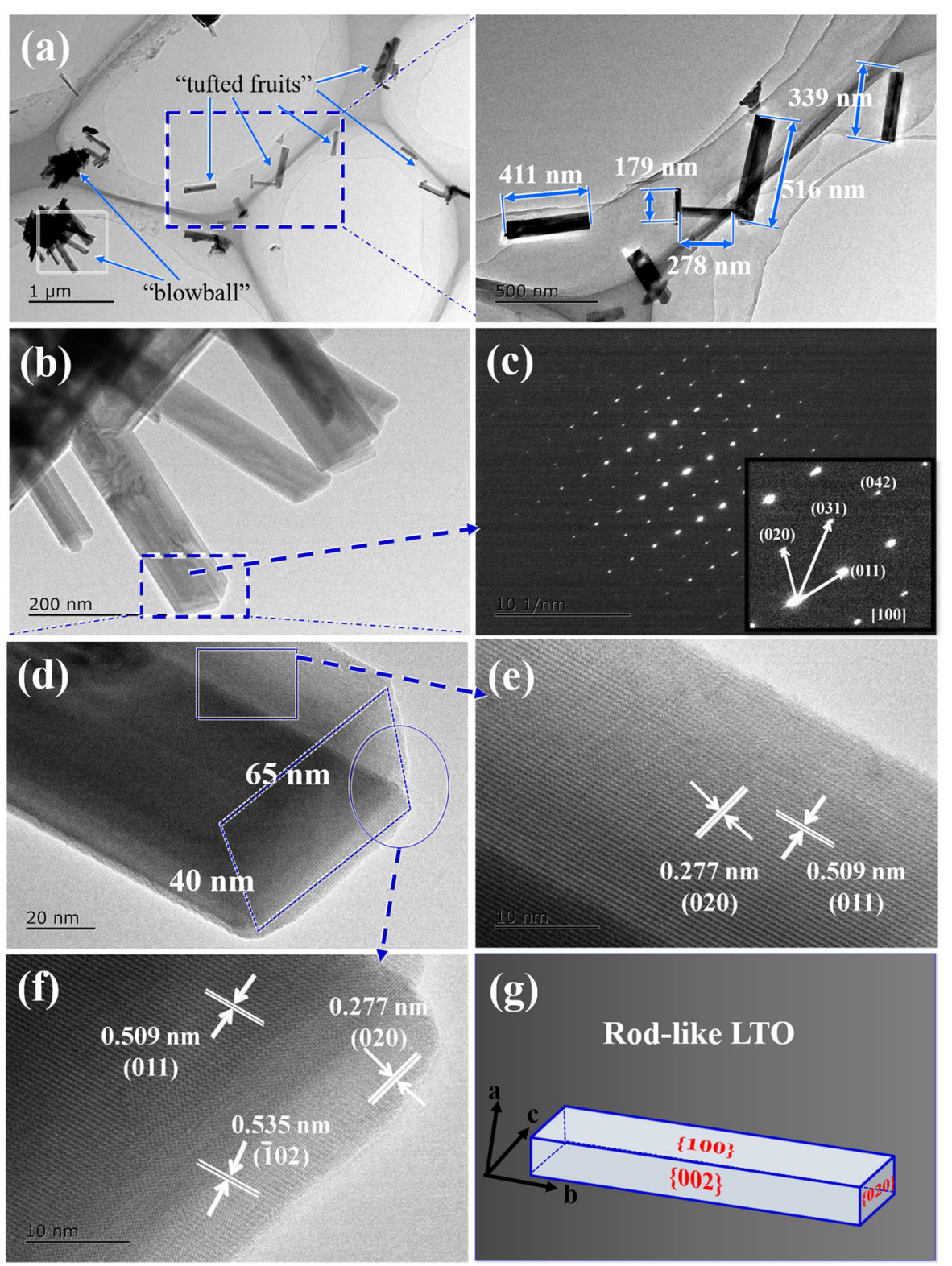

Fig. 3 TEM (a, b), SAED (c), and HRTEM (d-f) images and a morphology model $(\mathbf{g})$ of LTO NRs obtained at $700^{\circ} \mathrm{C}$ for $3 \mathrm{~h}$ in LiCl-KCl with a reactant:salt ratio of $1: 3$.

crystal plane with a spacing of $0.535 \mathrm{~nm}$ ascribed to the $(-102)$ plane, was detected. Combined with the HRTEM results in Fig. S6, these results confirmed that the two side facets of LTO NRs were $\{002\}$. Thus, the morphology model of LTO NRs, as schematized in Fig. 3g, was preferred along the [010] direction, with the $\{100\}$ and $\{002\}$ facets prominently exposed.

\section{Growth mechanism of dandelion-like LTO nanostructures}

As reported previously ${ }^{13-24}$, crystal growth in a liquid medium strongly relies on numerous factors, including reactant-solvent interactions (solubility and diffusion rate of the solute) and crystal-solvent interactions (diffusion rate and supersaturation of the targeted crystal). Because the eutectic temperature of the $\mathrm{LiCl}-\mathrm{KCl}$ binary salt is $\sim 300^{\circ} \mathrm{C}^{24}$, the liquid reaction medium is formed at $>300^{\circ} \mathrm{C}$. Thermodynamic calculations suggest that $\mathrm{La}_{2} \mathrm{O}_{3}$ can react with lithium chloride (reaction (4), $\Delta_{\mathrm{r}} G_{4}{ }^{*}=-30.9+$ $0.0494 \mathrm{~T}(\mathrm{~kJ} / \mathrm{mol}))$ at temperatures as low as room temperature, which resulted in good growth of $\mathrm{LaOCl}$ microplates with $\mathrm{LiCl}-\mathrm{KCl}$ as the molten salt (Fig. S8a and c) ${ }^{24}$. In the present work, $\mathrm{TiO}_{2}$ was also included and showed solubility in molten $\mathrm{LiCl}-\mathrm{KCl}$ (Fig. S8b, d). Figure 1 indicates that the $\mathrm{LaOCl}$ phase appeared in the samples 
fired at $400-600^{\circ} \mathrm{C}$, whereas it disappeared at $700-900{ }^{\circ} \mathrm{C}$. Moreover, as determined from the FE-SEM observations (Figs. S4 and S5), the addition of $\mathrm{TiO}_{2}$ led to the propagation of reaction (5) in a random and outward direction to the (001) facet of plate-like $\mathrm{LaOCl}$ crystallites ${ }^{24,36}$. From these results and the XRD results, we can conclude that $\mathrm{LaOCl}$ microplates were initially formed via reaction (4) and then acted as an intermediate substrate for the crystal growth of LTO via reaction (5). Note that the transformation from $\mathrm{LaOCl}$ to $\mathrm{LTO}$ is a pseudomorphic reaction because LTO with a monoclinic structure (Fig. S9a) does not have any motifs in common with the tetragonal $\mathrm{LaOCl}^{36}$. However, monoclinic $\mathrm{TiO}_{2}$ contains the same motif as a $\mathrm{TiO}_{6}$ octahedron (Fig. S9b). Thus, the transformation from $\mathrm{TiO}_{2}$ to LTO might be viewed as a topotactic reaction.

$$
\begin{aligned}
& 2 \mathrm{LiCl}+\mathrm{La}_{2} \mathrm{O}_{3}=2 \mathrm{LaOCl}+\mathrm{Li}_{2} \mathrm{O} \\
& 2 \mathrm{LaOCl}+\mathrm{Li}_{2} \mathrm{O}+2 \mathrm{TiO}_{2}=\mathrm{La}_{2} \mathrm{Ti}_{2} \mathrm{O}_{7}+2 \mathrm{LiCl}
\end{aligned}
$$

Several metal oxides with monoclinic structures have been synthesized in the form of $2 \mathrm{D}$ materials ${ }^{2,4-19,37-40}$. In perovskite-related crystals containing $\mathrm{TiO}_{6}$ octahedrons, such as LTO (Fig. S9a), only La-O bonds break along the interlayer of the (002) planes, whereas chains of $\mathrm{La}-\mathrm{O}$ and $\mathrm{Ti}-\mathrm{O}$ bonds must break when the surfaces are cleaved along the (100) and (020) planes. Based on the concept of a periodic bond chain $(\mathrm{PBC})^{15,16,38}$ and the broken bond model, the (002) plane along the interlayer of LTO should have the lowest surface energy and will preferentially develop in the equilibrium configuration. The layered crystal structure with weak bonding is considered crucial for $2 \mathrm{D}$ growth, consistent with the previously reported LTO prepared by MSS or other methods ${ }^{2,4-19}$, as LTO normally exhibits a plate-like structure. However, in the current work, the LTO crystal exhibited unnatural rodlike growth and formed a dandelion-like structure.

DFT calculations were performed to interpret the growth mechanism of LTO crystals. On the basis of the crystallization behaviors of the LTO plate ${ }^{2,4-16}$ (Fig. S10) and rod (Fig. 3g) structures, the surface energies on the (002), (100), and (020) planes of the LTO crystal were investigated. As listed in Table S2, in the intrinsic LTO crystal, the (002) plane has a lower surface energy $\left(0.51 \mathrm{~J} / \mathrm{m}^{2}\right)$ than the (100) $\left(1.29 \mathrm{~J} / \mathrm{m}^{2}\right)$ and $(020)\left(0.96 \mathrm{~J} / \mathrm{m}^{2}\right)$ planes. The calculation data are consistent with the PBC concept and the plate-like LTO structure ${ }^{13-19}$. By contrast, in the case of rod-like $\mathrm{LTO}$, the $\mathrm{LaOCl}$ acting as an intermediate substrate was involved in the synthesis (reactions (4) and (5)). We also separately carried out LTO syntheses in the single $\mathrm{LiCl}, \mathrm{KCl}$, and $\mathrm{NaCl}$ salts (the last two salts have been widely used in the synthesis of 2D LTO ${ }^{13-19}$, as shown in Figs. S11-S16). The results revealed that only the use of $\mathrm{LiCl}$ could form the $\mathrm{LaOCl}$ intermediate and achieve the growth of rod-like LTO structures, while the use of $\mathrm{KCl}$ led to the direct formation of granular or platelike LTO, and $\mathrm{NaCl}$ resulted in formation of the $\left(\mathrm{Na}_{0.5} \mathrm{La}_{0.5}\right) \mathrm{TiO}_{3}$ intermediate, which was converted to plate-like LTO at $\geq 1000{ }^{\circ} \mathrm{C}$. Notably, no dandelion-like (or rod-like) LTO structure was observed in $\mathrm{KCl}$ or $\mathrm{NaCl}$ alone. These results indicated that the $\mathrm{LiCl}$ salt plays a dominant role in the formation of dandelion-like structures.

DFT calculations were also conducted when the salt was absorbed on the planes of the LTO crystal. The optimized adsorption configurations and calculated surface and adsorption energies are shown in Fig. 4a and Table S2, respectively. The calculation results (Fig. 5a) show that after salt adsorption, the surface energies all decrease. Although the (002) plane shows the greatest rate of decrease, it still has much lower surface energies (0.26, 0.17 and $0.17 \mathrm{~J} / \mathrm{m}^{2}$ for $\mathrm{LiCl}, \mathrm{KCl}$, and $\mathrm{NaCl}$, respectively; see Table S2) than the $(100)(1.11,1.06$, and $\left.1.05 \mathrm{~J} / \mathrm{m}^{2}\right)$ and $(020)\left(0.92,0.84\right.$, and $\left.0.85 \mathrm{~J} / \mathrm{m}^{2}\right)$ planes. By comparison, under $\mathrm{LiCl}$ adsorption, the rate of decrease of the surface energy on the (100) plane $(13.95 \%$, indicated by the black arrow in Fig. 5a) is more significant than that on the $(020)$ plane $(4.17 \%$, indicated by the green arrow), but this distinction is not evident under the adsorption of $\mathrm{KCl}$ or $\mathrm{NaCl}$. Moreover, DFT calculations reveal that the $\mathrm{LiCl}$ adsorption energy on the LTO $(020)$ plane $(-94.30 \mathrm{~kJ} / \mathrm{mol}$, indicated by the blue arrow in Fig. 5b) is obviously lower than those on the (002) and (100) planes $(-134.20$ and $-137.10 \mathrm{~kJ} / \mathrm{mol}$, respectively), whereas both $\mathrm{KCl}$ and $\mathrm{NaCl}$ have similar adsorption energies on these three planes. Based on these results, it is reasonable to conclude that $\mathrm{LiCl}$ has a more distinct effect on the (100) plane than on the (020) plane. In other words, the stronger adsorption of $\mathrm{LiCl}$ on the (100) plane could greatly restrain the growth along the [100] axis, correspondingly resulting in LTO growth along the [010] axis (Fig. 4b). Therefore, LTO exhibits a rod-like shape that assembles into a dandelion-like structure.

In the present study, it is worth mentioning that although the role of salt was discussed based on DFT calculations, the exact mechanism for dandelion-like LTO formation is still difficult to determine because there is no available method for routinely studying crystal growth in situ in a salt medium. However, based on the present results in Figs. 1-5 and S1-S16 and the results of related studies $^{14-24}$, a plausible growth process of LTO dandelion nanostructures (shown in Fig. 6) could be proposed as follows: (1) At $300-400{ }^{\circ} \mathrm{C}, \mathrm{La}_{2} \mathrm{O}_{3}$ dissolves in a salt medium and then homogeneously mixes with chlorine ions. Reaction (4) proceeds rapidly to form $\mathrm{LaOCl}$. Once the as-formed $\mathrm{LaOCl}$ is oversaturated in the salt medium, 
(a)

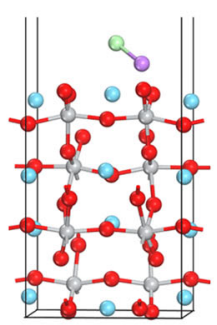

(002)

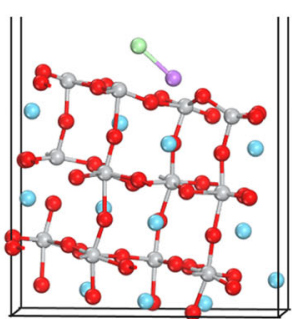

(100)

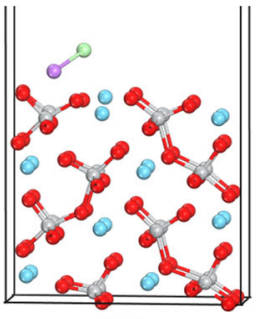

(020)
○ La

- Ti

- 0

○ $\mathbf{C l}$

○ Li/K/Na

(b)

Plate-like LTO

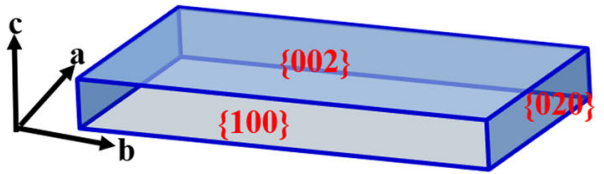

Rod-like LTO

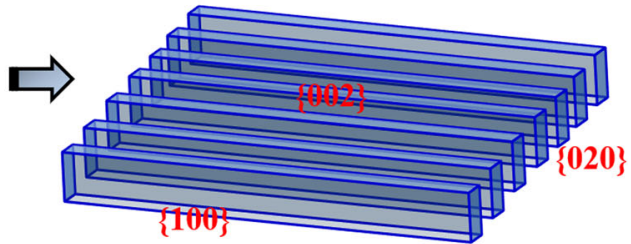

Fig. 4 The configurations (a) of salts absorbed on the (002), (100), and (020) planes of LTO and a schematic illustration (b) of structural conversion of LTO from plate to rod.
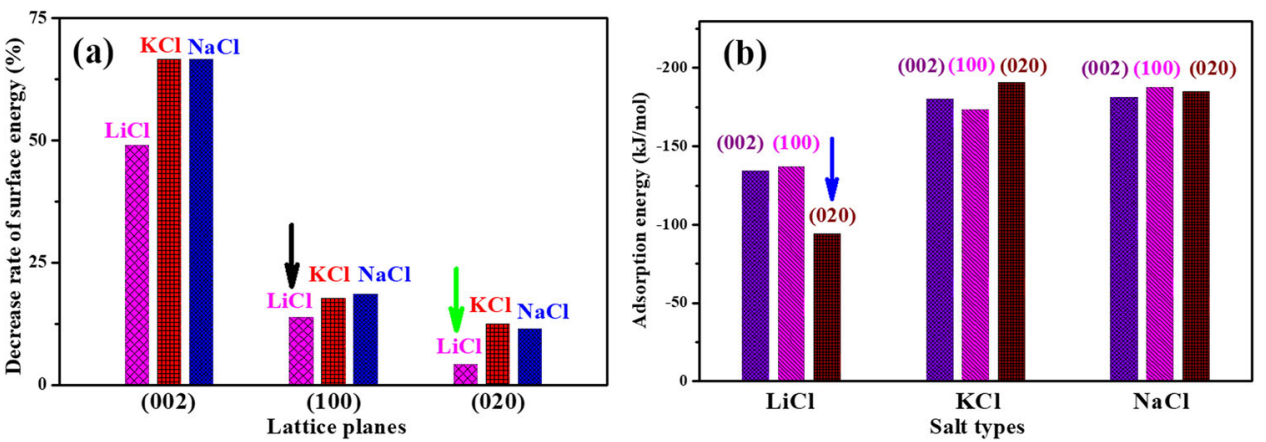

Fig. 5 The rates of decrease in the surface energy (a) and absorption energy (b) of $\mathrm{LiCl}, \mathrm{KCl}$, and $\mathrm{NaCl}$ for the (002), (100), and (020) planes.

the $\mathrm{LaOCl}$ plate is generated with the $\{001\}$ facet exposed. When the topmost-exposed layers of the $\{001\}$ facet are $\mathrm{Cl}$ and La atoms, it is chemically nonequivalent ${ }^{36}$. The very small dissolved $\mathrm{TiO}_{2}$ particles (probably in a $\mathrm{TiO}_{6}$ motif) diffuse to the $\{001\}$ facet of $\mathrm{LaOCl}$, and the exchange of chlorine ions to $\mathrm{TiO}_{6}$ occurs rapidly, as shown in Fig. 6(I) and (a). (2) At $400-700{ }^{\circ} \mathrm{C}$, the $\mathrm{TiO}_{6}$ octahedra are transformed into $\mathrm{LaOCl}$ crystals by the topotactic reaction, and subsequently, LTO germination occurs on the (001) surface of $\mathrm{LaOCl}$ via reaction (5). As shown in Fig. 6 (II), LTO is oriented randomly on the LaOCl lamellar structure for NR-like growth. This reaction starts from the basal surface and further propagates along the [010] direction (under the effect of $\mathrm{LiCl}$ salt) until the $\mathrm{LaOCl}$ substrate is completely consumed (Fig. 5b). 3) At $\geq 700{ }^{\circ} \mathrm{C}$, the high temperature drives the above steps, and an LTO "dandelion" is formed, which is composed of the randomly ordered LTO NRs (Fig. 6(III) and (c)). In agreement with this mechanism, we also found that pure $\mathrm{LaOCl} / \mathrm{LTO}$ and $\mathrm{LTO} / \mathrm{TiO}_{2}$ composites could be obtained by controlling the ratio of $\mathrm{TiO}_{2}$ and $\mathrm{La}_{2} \mathrm{O}_{3}$ (Fig. S17).

\section{Photocatalytic performance of dandelion-like LTO nanostructures}

To investigate the photocatalytic performance of dandelion-like LTO and elucidate the morphology-activity relationship, the photodegradation behavior of $\mathrm{RhB}$ $(10 \mathrm{mg} / \mathrm{ml})$ was evaluated over LTO with dandelion- and plate-like morphologies under UV light irradiation. The plate-like LTO was prepared by MSS with $\mathrm{NaCl}$ as the molten salt at $1100^{\circ} \mathrm{C}$ (Figs. S15 and S16b). In the dark, the concentrations of $\mathrm{RhB}$ showed no change in all samples, as shown in Fig. 7a. After irradiation with UV light for 

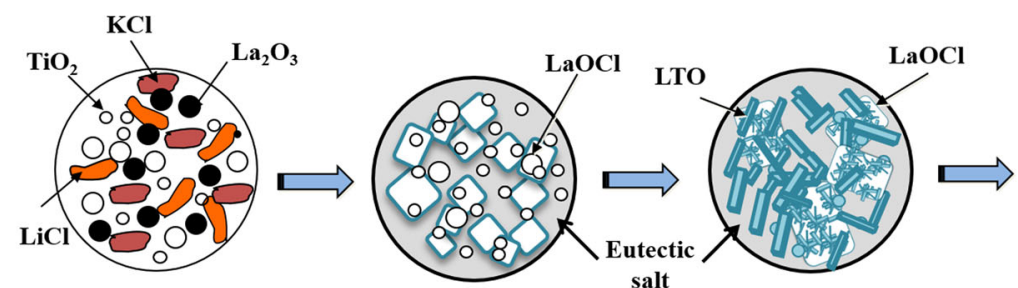

salt

Room temperature

$300^{\circ} \mathrm{C}<\mathrm{T} \leq 400^{\circ} \mathrm{C}$

$400^{\circ} \mathrm{C}<\mathrm{T}<7^{\circ} 0^{\circ} \mathrm{C}$

(II)

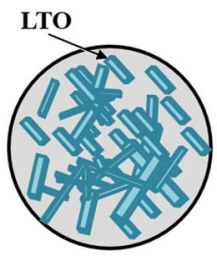

$\mathrm{T} \geq 700^{\circ} \mathrm{C}$

(I)

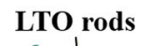

(II)

(III)

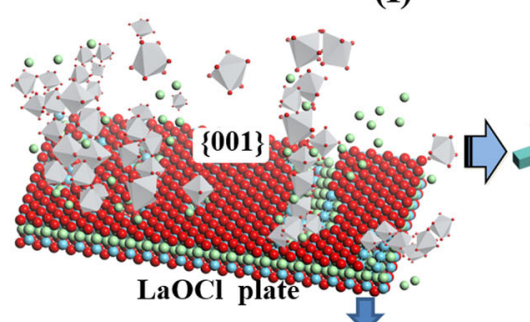

LTO rods

$\checkmark$

(020)

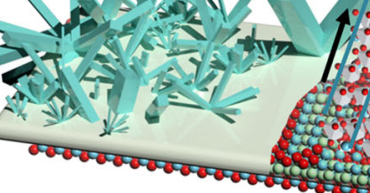

$\checkmark$
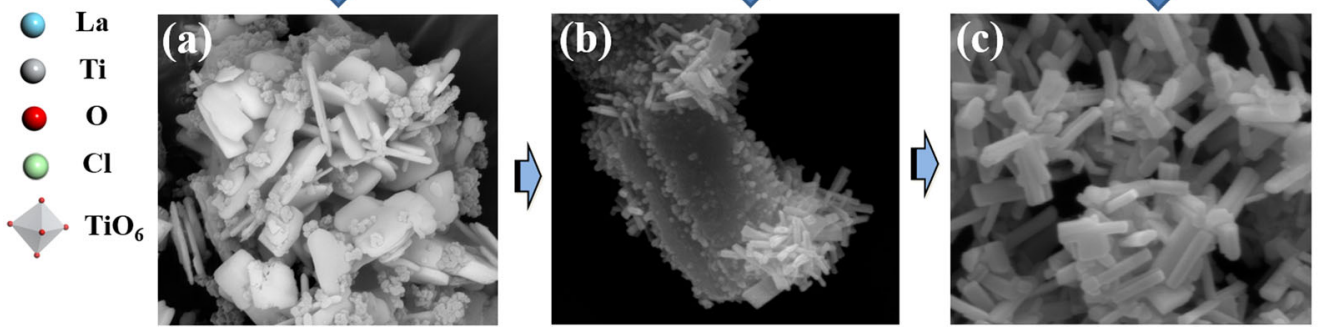

Fig. 6 A schematic of the MSS process of dandelion-like LTO nanostructure formation in LiCl-KCl. ((I) A LaOCl plate projected along the [100] or [010] direction (ICDD 89-6883) and (II) an LTO rod projected along the [010] direction (ICDD 81-1066)).
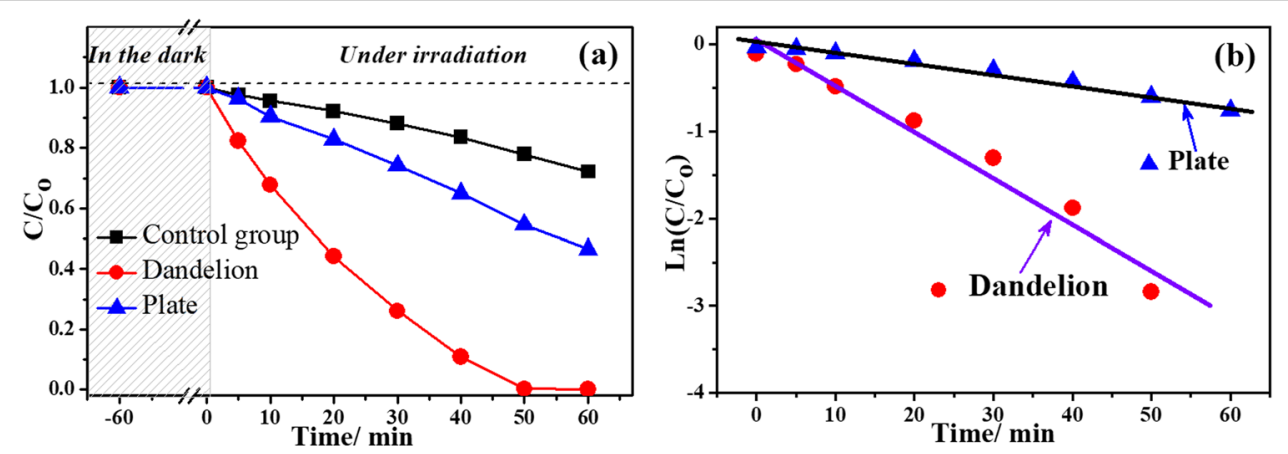

Fig. 7 Photocatalytic degradation curves (a) of the RhB aqueous solution and $L n$ (relative concentration) as a function of time (b) over dandelion-like (fired at $700{ }^{\circ} \mathrm{C}$ in $\mathrm{LiCl}-\mathrm{KCl}$ with a reactant:salt ratio of 1:3) and plate-like (fired at $1100^{\circ} \mathrm{C}$ in $\mathrm{NaCl}$ with a reactant:salt ratio of 1:3) LTO under UV light irradiation.

$60 \mathrm{~min}$, the plate-like LTO removed only $\sim 20 \%$ of $\mathrm{RhB}$ (excluding the RhB self-degradation of $\sim 28 \%$ ). In contrast, the dandelion-like LTO completely degraded the RhB in $50 \mathrm{~min}$. The photocatalytic degradation reaction kinetics (Fig. 7b) were quantified according to the pseudo-firstorder Eq. (1), showing that the kinetic rate of using dandelion-like LTO $\left(k, 3.1 \mathrm{~h}^{-1}\right.$, correlation coefficient of $\left.R^{2}>0.97\right)$ is approximately 5 times higher than that using plate-like LTO $\left(k, 0.63 \mathrm{~h}^{-1}\right.$, correlation coefficient of $R^{2}>$ 0.99). In addition, the dandelion-like LTO also possesses much better photocatalytic ability for $\mathrm{RhB}$ degradation than most reported plate-like LTO structures (summarized in Table S3), further verifying the superiority of the current structure. These results indicated that the dandelion-like nanostructure can significantly enhance the photocatalytic activity. 


\section{Origin of enhanced photocatalytic performance on dandelion-like LTO nanostructures}

As mentioned above, dandelion-like LTO is composed of LTO NRs, in which the axial size is much larger than the radial size, so the $\{100\}$ and $\{002\}$ facets are predominantly exposed in LTO NRs (Fig. 3g). By contrast, because the width of plate-like LTO is larger than its thickness, the $\{002\}$ facet could be considered the major exposed area (Figs. $4 \mathrm{~b}$ and S10). To determine the origin of enhanced photocatalytic performance on dandelionlike LTO, additional characterizations and DFT calculations were carried out.

UV-vis DRS was first performed to experimentally measure the energy band of dandelion- and plate-like LTO. Figure S18 shows that the derived electronic band gaps of dandelion- and plate-like LTO were $\sim 3.37$ and $3.25 \mathrm{eV}$, respectively. Notably, an absorption shoulder in the visible region was observed in spectrum of plate-like LTO, which might be attributed to the predominant $\{002\}$ surface incorporating oxygen vacancies, as shown in Fig. S19. Ordinarily, a narrower band gap results in higher photocatalytic activity ${ }^{26}$. However, in this work, the dandelion-like LTO with a slightly wider band gap had much better photodegradation behavior than the plate-like LTO. Therefore, this finding precludes the possibility that the distinct photocatalysis between these two LTO morphologies originated from their intrinsic band gap. The nitrogen adsorption isotherms of LTO are given in Fig. S20. The BET surface area of the dandelion-like LTO was $17.5 \mathrm{~m}^{2} / \mathrm{g}$, but that of the platelike LTO was only $2.5 \mathrm{~m}^{2} / \mathrm{g}$. The higher surface area of the former might be explained by the large increase in the $\{100\}$ facets in rod-like LTO (Fig. 4b), which may also increase the photocatalytic activity. However, the adsorption-desorption equilibrium in dark conditions showed no difference (Fig. 7a). Thus, it is reasonable to believed that the surface area might not be the dominant reason for the enhanced photocatalytic activity of dandelion-like $\mathrm{LTO}^{17-19}$.

XPS was performed to further characterize the chemical state and composition of dandelion- and plate-like LTO surfaces. The full spectrum (Fig. 8a) shows the elements $\mathrm{La}, \mathrm{O}, \mathrm{Ti}$, and $\mathrm{C}$ in both samples. The corresponding high-resolution XPS spectra of La 3d, O 1s, and Ti 2p are presented in Fig. 8b-d, respectively. In Fig. 8b, the La 3d spectra of these two LTO structures showed the same doublet peaks of the $3 \mathrm{~d}_{2 / 5}$ and $3 \mathrm{~d}_{3 / 2}$ states of $\mathrm{La}^{3+}$, indicating the constant La arrangement in the surface of the dandelion- and plate-like structures ${ }^{39,40}$. Figure $8 \mathrm{c}$ reveals that the plate-like LTO exhibited symmetric Ti $2 \mathrm{p}_{1 / 2}$ and $2 \mathrm{p}_{3 / 2}$ peaks at 460.9 and $455.1 \mathrm{eV}$, respectively, which could be assigned to the $\mathrm{Ti}-\mathrm{O}$ bonds of $\mathrm{TiO}_{6}$ octahedral slabs. By contrast, the Ti 2p peaks of dandelion-like LTO were asymmetric and deconvoluted into two components. The additional peaks at 462.7 and $456.5 \mathrm{eV}$ were assigned to the unsaturated $\mathrm{Ti}$ of $\mathrm{TiO}_{5}$ motifs on the unsaturated Ti-terminated facet ${ }^{17}$, which might be indicative of the largely increased $\{100\}$ facets on LTO NRs (Fig. 4b). In
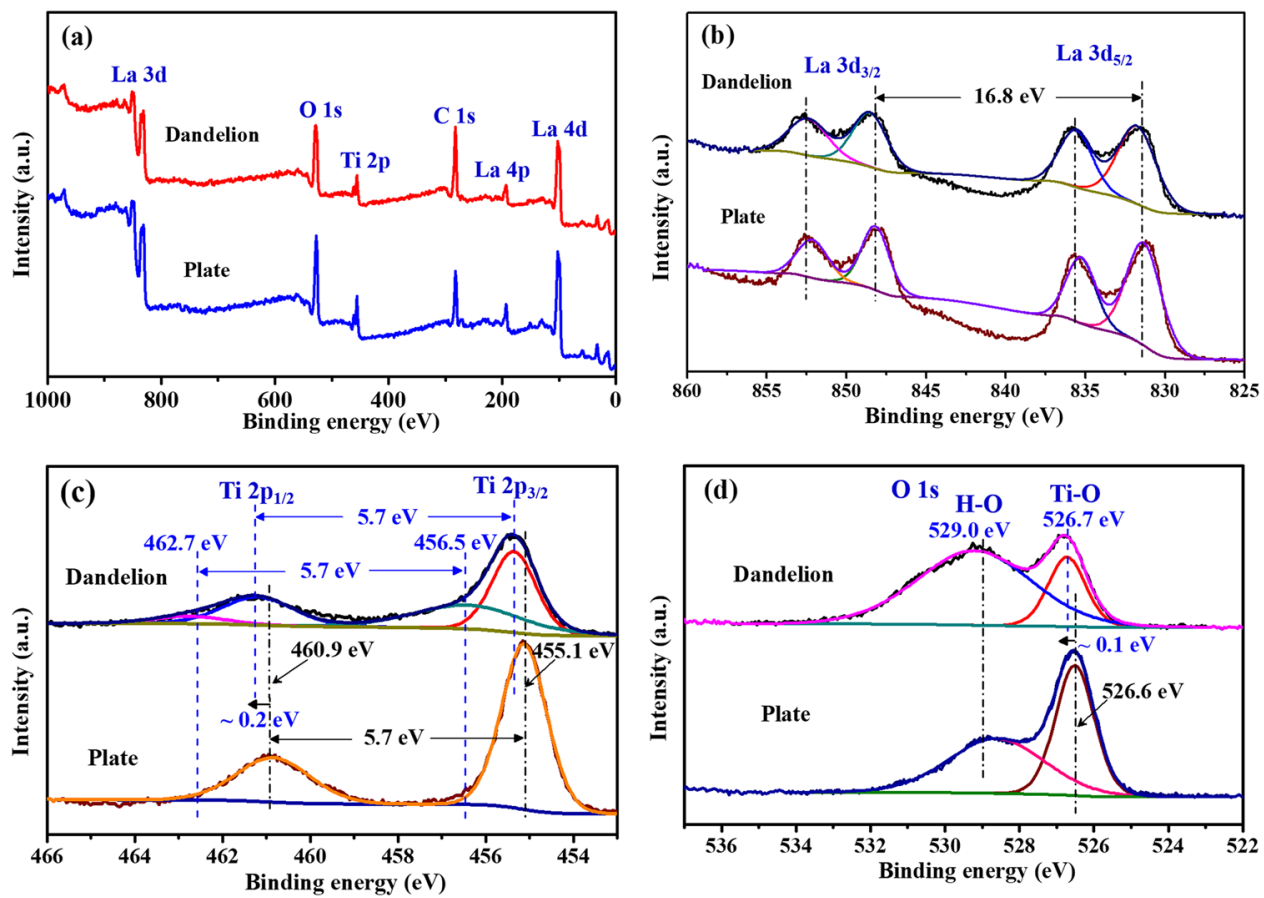

Fig. 8 XPS wide-scan spectrum (a) and high-resolution XPS spectra of La 3d (b), Ti 2p (c) and O 1s (d) of dandelion- and plate-like LTO. 
particular, the $\mathrm{Ti} 2 \mathrm{p}$ peak of the $\mathrm{TiO}_{6}$ slabs in dandelionlike LTO showed a higher binding energy of $\sim 0.2 \mathrm{eV}$ than that in the plate-like LTO. A similar shift for the O $1 \mathrm{~s}$ spectrum (Fig. 8d) was also detected, in which both dandelion- and plate-like LTO gave rise to two typical O $1 \mathrm{~s}$ peaks, which were assigned to the physisorption of $\mathrm{H}-\mathrm{O}(529.0 \mathrm{eV}$; note that the relative integral of $\mathrm{H}-\mathrm{O}$ in the dandelion-like LTO was obviously larger than that in the plate-like LTO, suggesting a significant increase in unsaturated Ti-terminated $\{100\}$ facets, which adsorb the $\mathrm{OH}^{-}$from water ${ }^{17-19}$ ) and $\mathrm{Ti}-\mathrm{O}$ bonds. The $\mathrm{Ti}-\mathrm{O}$ peak position for the dandelion-like LTO was at $526.7 \mathrm{eV}$, whereas that for the plate-like LTO was at $526.6 \mathrm{eV}$, showing a positive shift $(\sim 0.1 \mathrm{eV})$. These shifts suggested that the electron density of partial LTO surfaces decreased when the structure changed from plate to dandelion. Electron transfer between successive $\{002\}$ and $\{100\}$ facets was probably the reason for the prominent exposed $\{002\}$ and $\{100\}$ facets of LTO NRs.
DFT calculations were carried out to obtain insight into the effect of morphology and to explain the enhanced photocatalytic activity of LTO NRs with coexposed $\{002\}$ and $\{100\}$ facets. The optimized geometrical structures of the $\{002\}$ and $\{100\}$ facets are illustrated in Fig. S21; notably, the electronic structure of the $\{020\}$ facet shown in Fig. S22 was similar to that of the $\{002\}$ facet, and considering the relatively low proportion of the former in LTO rods (Fig. 4b), the following discussion is focused on the $\{002\}$ facet. In particular, the $\{100\}$ facet features very long $\mathrm{Ti}-\mathrm{O}-\mathrm{Ti}$ bonds, which can lead to destabilization of the $2 p$ states on the surface $O$ atoms and thus the high reactivity of the $\{100\}$ facet $^{27,41}$. The electronic structures of the $\{002\}$ and $\{100\}$ facets, determined by DFT calculations, are presented in Fig. 9a. These results reveal that the Fermi level of the $\{002\}$ facet is fixed at the top of the $\mathrm{VB}$, whereas because of the presence of the destabilized $\mathrm{O}$ $2 \mathrm{p}$ states (indicated by the red dotted circle), the Fermi level of $\{100\}$ should be more positive (indicated by the

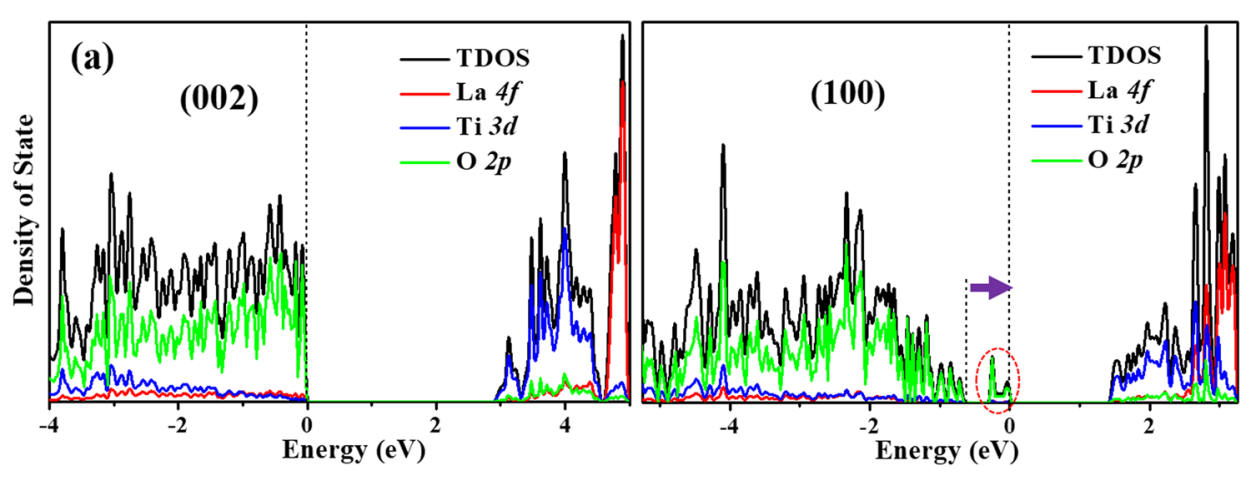

(b)

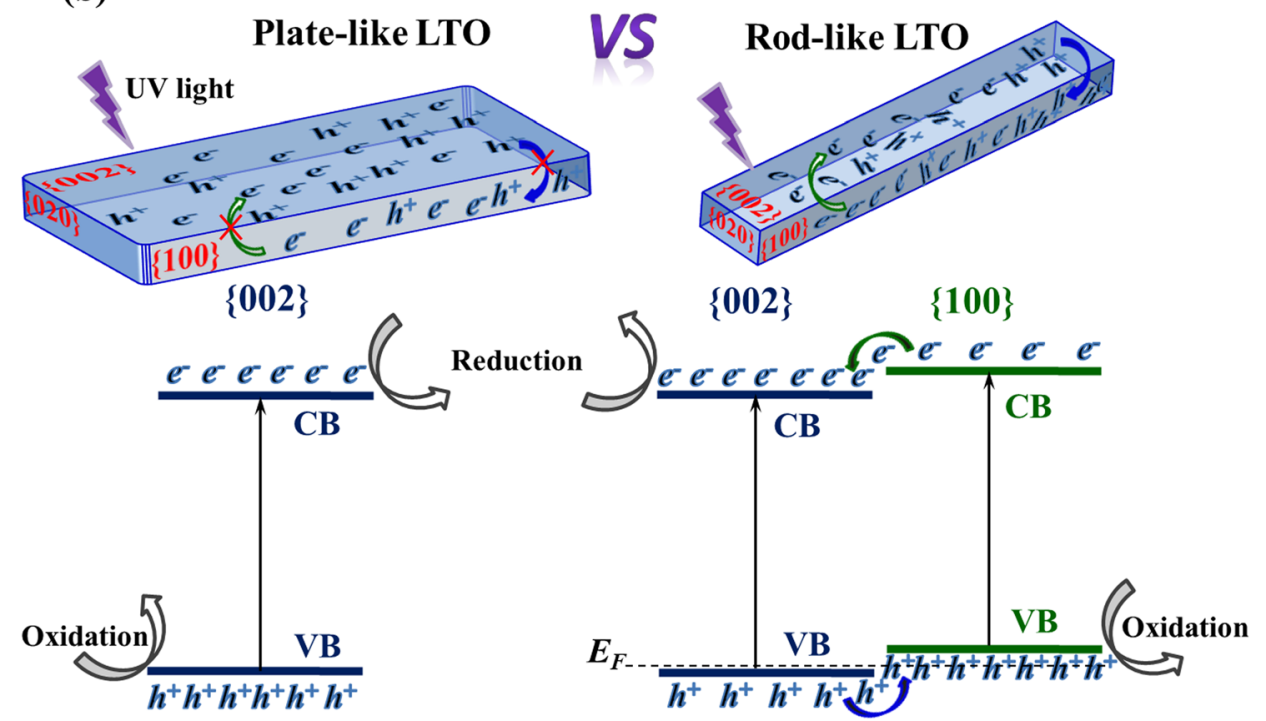

Fig. 9 Density of state (DOS) plots (a) for the $\{002\}$ and $\{100\}$ facets of LTO. TDOS, La 4f, Ti 3d and O 2p are the total DOS and partial DOS of La 4f, Ti 3d and $\mathrm{O} 2 \mathrm{p}$, respectively. Schematic illustration $(\mathbf{b})$ of the enhanced photocatalysis on the dandelion-like nanostructure. 
purple arrow). This means that the band gap of the $\{100\}$ facet becomes narrow, and the Fermi levels of the $\{002\}$ and $\{100\}$ facets are not equivalent ${ }^{17,27}$. Notably, such a calculated band structure is consistent with the direct measurements of the flat-band potential $\left(V_{\mathrm{fb}}\right)$ shown in Fig. S23. However, these two facets are successive (Fig. $3 \mathrm{~g})$. The different Fermi levels and density of state (DOS) values reveal that the migration and separation of photogenerated electron-hole pairs can occur between the $\{002\}$ and $\{100\}$ surfaces of LTO, which might also contribute to the shifts in the XPS results (Fig. 8).

On the basis of the above characterizations and DFT calculations, a "surface heterojunction" within LTO NRs, which is considered the origin of the enhanced photocatalytic activity of dandelion-like LTO, was proposed to be formed with the main coexposed $\{002\}$ and $\{100\}$ facets, as shown in Fig. 9b. Under UV light irritation, the VB electrons in LTO were excited to the conduction band (CB) level, and the holes remained at the VB level. In the platelike LTO, the majority of photogenerated electron-hole pairs were collectively accumulated on the main exposed $\{002\}$ facets, which resulted in the easy recombination of electrons and holes, and only a fraction of electron-hole pairs could participate in the photocatalytic reaction. By contrast, for the rod-like LTO with the $\{002\}$ and $\{100\}$ facets coexposed, the electrons and holes could efficiently separate and transfer to the $\{002\}$ and $\{100\}$ facets, respectively. Therefore, the $\{002\}$ facets could act as reduction sites (which could be further confirmed by the photoreduction of Pt particles ${ }^{42}$ shown in Fig. S24), and the $\{100\}$ facets acted as oxidation sites on the LTO NRs. As a result, the dandelion-like LTO, composed mainly of LTO NRs, could efficiently improve the photocatalytic performance compared with the plate-like LTO. The separation efficiency of photogenerated electrons and holes for the dandelion- and plate-like LTO could be further verified by the photocurrent responses (Fig. S25) and photoluminescence spectra (Fig. S26).

\section{Conclusions}

The molten salt technique was developed to synthesize dandelion-like LTO nanostructures from inexpensive raw materials of $\mathrm{La}_{2} \mathrm{O}_{3}$ and $\mathrm{TiO}_{2}$. The lowest synthesis temperature of $700^{\circ} \mathrm{C}$ is much lower than those reported for other techniques. $\mathrm{LaOCl}$ microplates were formed as the substrate for further growth of dandelion-like LTO nanostructures. DFT calculations indicated the presence of a strong interaction between $\mathrm{LiCl}$ salt and the (100) plane, resulting in the preferred growth of LTO along the [010] axis and the subsequent formation of LTO NRs. In addition, a surface heterojunction within the coexposed $\{002\}$ and $\{100\}$ facets was observed in the LTO NRcontaining dandelion nanostructure. The efficient migration and separation of photogenerated electrons and holes between the $\{002\}$ and $\{100\}$ facets of LTO NRs resulted in enhanced photocatalytic performance. This work not only describes a new dandelion-like LTO nanostructure but also highlights the design of nanomaterials with distinct exposed facets by the molten salt method, providing new possibilities to explore structure-activity relationships.

\section{Acknowledgements \\ This work was supported by the National Natural Science Foundation of China (Grant Nos 51672194, 51702241, and 51872210), the Program for Innovative Teams of Outstanding Young and Middle-aged Researchers in the Higher Education Institutions of Hubei Province (T201602) and the Key Program of Natural Science Foundation of Hubei Province, China (Contract No 2017CFA004) \\ Author details \\ ${ }^{1}$ The State Key Laboratory of Refractories and Metallurgy, Wuhan University of Science and Technology, Wuhan 430081, China. ${ }^{2}$ Henan Key Laboratory of High Temperature Functional Ceramics, Zhengzhou University, Zhengzhou 450052, China. ${ }^{3}$ College of Engineering, Mathematics and Physical Sciences, University of Exeter, Exeter EX4 4QF, UK}

Conflict of interest

The authors declare that they have no conflict of interest.

\section{Publisher's note}

Springer Nature remains neutral with regard to jurisdictional claims in published maps and institutional affiliations.

Supplementary information is available for this paper at https://doi.org/ 10.1038/s41427-019-0194-y.

Received: 13 August 2019 Revised: 17 November 2019 Accepted: 20 November 2019.

Published online: 24 January 2020

\section{References}

1. Ishizawa, N., Marumo, F., Iwai, S., Kimura, M. \& Kawamura, T. Compounds with perovskite-type slabs. $\mathrm{V}$. A high-temperature modification of $\mathrm{La}_{2} \mathrm{Ti}_{2} \mathrm{O}_{7}$. Acta Crystallogr. B 38, 368-372 (1982).

2. Yan, H., Ning, H., Kan, Y., Wang, P. \& Reece, M. J. Piezoelectric ceramics with super-high curie points. J. Am. Ceram. Soc. 92, 2270-2275 (2009).

3. Lu, Y. et al. Reactive sputtering deposition of perovskite oxide and oxynitride lanthanum titanium films: structural and dielectric characterization. Cryst. Growth Des. 13, 4852-4858 (2013).

4. Wang, Z., Teramura, K., Hosokawa, S. \& Tanaka, T. Photocatalytic conversion of $\mathrm{CO}_{2}$ in water over Ag-modified $\mathrm{La}_{2} \mathrm{Ti}_{2} \mathrm{O}_{7}$. Appl. Catal. B: Environ. 163, 241-247 (2015).

5. Kim, J. et al. Nickel-loaded $\mathrm{La}_{2} \mathrm{Ti}_{2} \mathrm{O}_{7}$ as a bifunctional photocatalyst. Chem. Commun. 21, 2488-2489 (2002).

6. Hwang, D. W. et al. Photocatalytic degradation of $\mathrm{CH}_{3} \mathrm{Cl}$ over a nickel-loaded layered perovskite. Ind. Eng. Chem. Res. 42, 1184-1189 (2003).

7. Arney, D., Porter, B., Greve, B. \& Maggard, P. A. New molten-salt synthesis and photocatalytic properties of $\mathrm{La}_{2} \mathrm{Ti}_{2} \mathrm{O}_{7}$ particles. J. Photochem. Photobiol. A 199, 230-235 (2008).

8. Abe, R., Higashi, M., Sayama, K., Abe, Y. \& Sugihara, H. Photocatalytic activity of $\mathrm{R}_{3} \mathrm{MO}_{7}$ and $\mathrm{R}_{2} \mathrm{Ti}_{2} \mathrm{O}_{7}(\mathrm{R}=\mathrm{Y}, \mathrm{Gd}, \mathrm{La} ; \mathrm{M}=\mathrm{Nb}, \mathrm{Ta})$ for water splitting into $\mathrm{H}_{2}$ and $\mathrm{O}_{2}$. J. Phys. Chem. B 110, 2219-2226 (2006).

9. Li, K., Wang, Y., Wang, H., Zhu, M. \& Yan, H. Hydrothermal synthesis and photocatalytic properties of layered La2Ti2O7 nanosheets. Nanotechnology 17, 4863 (2006).

10. Song, H., Peng, T., Cai, P., Yi, H. \& Yan, C. Hydrothermal synthesis of flaky crystallized $\mathrm{La}_{2} \mathrm{Ti}_{2} \mathrm{O}_{7}$ for producing hydrogen from photocatalytic water splitting. Catal. Lett. 113, 54-58 (2007). 
11. Kawashima, K. et al. An $\mathrm{NH}_{3}$-assisted flux-mediated direct growth of $\mathrm{LaTiO}_{2} \mathrm{~N}$ crystallites for visible-light-induced water splitting. J. Phys. Chem. C. 119, 15896-15904 (2015).

12. Feng, J. et al. Highly photo-responsive $\mathrm{LaTiO}_{2} \mathrm{~N}$ photoanodes by improvement of charge carrier transport among film particles. Adv. Funct. Mater. 24, 3535-3542 (2014)

13. Wang, Q., Hisatomi, T., Moriya, Y., Maeda, K. \& Domen, K. Physicochemical properties and photocatalytic $\mathrm{H}_{2}$ evolution activity of Rh-doped $\mathrm{La}_{2} \mathrm{Ti}_{2} \mathrm{O}_{7}$ prepared by molten salt synthesis. Catal. Sci. Technol. 3, 2098-2103 (2013).

14. Wagata, $\mathrm{H}$. et al. Chloride flux growth of $\mathrm{La}_{2} \mathrm{Ti}_{2} \mathrm{O}_{7}$ crystals and subsequent nitridation to form LaTiO 2 N crystals. Cryst. Growth Des. 15, 124-128 (2014).

15. Hojamberdiev, M., Yamaguchi, A., Yubuta, K., Oishi, S. \& Teshima, K. Fabrication of $\mathrm{La}_{2} \mathrm{Ti}_{2} \mathrm{O}_{7}$ crystals using an alkali-metal molybdate flux growth method and their nitridability to form LaTiO2N crystals under a high-temperature $\mathrm{NH} 3$ atmosphere. Inorg. Chem. 54, 3237-3244 (2015).

16. Orum, A. K. et al. Atomic force microscopy surface analysis of layered perovskite $\mathrm{La}_{2} \mathrm{Ti}_{2} \mathrm{O}_{7}$ particles grown by molten flux method. Jpn. J. Appl. Phys. 55, 08NB08 (2016).

17. Cai, X. et al. Charge separation in a nanostep structured perovskite-type photocatalyst induced by successive surface heterojunctions. J. Mater. Chem. A 5, 10442-10449 (2017).

18. Cai, $\mathrm{X}$. et al. Au nanorod photosensitized $\mathrm{La}_{2} \mathrm{Ti}_{2} \mathrm{O}_{7}$ nanosteps: successive surface heterojunctions boosting visible to near-infrared photocatalytic $\mathrm{H}_{2}$ evolution. ACS Catal. 8, 122-131 (2017)

19. Mao, L., Cai, X., Yang, S., Han, K. \& Zhang, J. Black phosphorus-CdS-La ${ }_{2} \mathrm{Ti}_{2} \mathrm{O}_{7}$ ternary composite: effective noble metal-free photocatalyst for full solar spectrum activated $\mathrm{H}_{2}$ production. Appl. Catal. B: Environ. 242, 441-448 (2019).

20. Huang, Z. et al. Molten salt synthesis of $\mathrm{La}_{2} \mathrm{Zr}_{2} \mathrm{O}_{7}$ ultrafine powders. Ceram. Int 42, 6221-6227 (2016)

21. Huang, Z., Duan, H., Liu, J. \& Zhang, H. Preparation of lanthanum cerate powders via a simple molten salt route. Ceram. Int. 42, 10482-10486 (2016).

22. Huang, Z. et al. Preparation of $\mathrm{CaZrO}_{3}$ powders by a microwave-assisted molten salt method. J. Ceram. Soc. Jpn. 124, 593-596 (2016).

23. Huang, Z. et al. Low temperature molten salt preparation of molybdenum nanoparticles. Int. J. Refract. Met. H. 54, 315-321 (2016).

24. Huang, Z., Zhang, H. \& Zhang, S. Growth of well-developed LaOCl microplates by chloride salt-assisted method. CrystEngComm 19, 2971-2976 (2017).

25. Yang, $\mathrm{H}$. G. et al. Antase $\mathrm{TiO} 2$ single crystals with a large percentage of reactive facets. Nature 453, 638-641 (2008).

26. Lin, R. et al. Quantitative study of charge carrier dynamics in well-defined $\mathrm{WO}_{3}$ nanowires and nanosheets: insight into the crystal facet effect in photocatalysis. J. Am. Chem. Soc. 140, 9078-9082 (2018).
27. Yu, J., Low, J., Xiao, W., Zhou, P. \& Jaroniec, M. Enhanced photocatalytic $\mathrm{CO}_{2}$ reduction activity of anatase $\mathrm{TiO}_{2}$ by coexposed $\{001\}$ and $\{101\}$ facets. J. Am. Chem. Soc. 136, 8839-8842 (2014).

28. Liu, X., Dong, G., Li, S., Lu, G. \& Bi, Y. Direct observation of charge separation on anatase $\mathrm{TiO}_{2}$ crystals with selectively etched $\{001\}$ facets. J. Am. Chem. Soc. 138, 2917-2920 (2016)

29. Kresse, G. \& Furthmüller, J. Efficient iterative schemes for ab initio total-energy calculations using a plane-wave basis set. Phys. Rev. B 54, 11169 (1996).

30. Perdew, J. P., Burke, K. \& Ernzerhof, M. Generalized gradient approximation made simple. Phys. Rev. Lett. 77, 3865-3868 (1996).

31. Gu, Y. et al. Nitridation of silicon powders catalyzed by cobalt nanoparticles. J. Am. Ceram. Soc. 98, 1762-1768 (2015).

32. Vanderbilt, $D$. Soft self-consistent pseudopotentials in a generalized eigenvalue formalism. Phys. Rev. B 41, 7892-7895 (1990).

33. Tafreshi, S. S., Roldan, A, Dzade, N. Y. \& de Leeuw, N. H. Adsorption of hydrazine on the perfect and defective copper (111) surface: A dispersioncorrected DFT study. Surf. Sci. 622, 1-8 (2014).

34. Zhou, L., Xiu, F., Qiu, M., Xia, S. \& Yu, L. The adsorption and dissociation of water molecule on goethite (010) surface: a DFT approach. Appl. Surf. Sci. 392, 760-767 (2017)

35. Gu, M., Wang, Z., Wang, C. \& Zheng, J. Atomic scale study of surface orientations and energies of $\mathrm{Ti}_{2} \mathrm{O}_{3}$ crystals. Appl. Phys. Lett. 111, 181603 (2017).

36. Afanasiev, P., Aouine, M., Deranlot, C. \& Epicier, T. Ordered arrays of nanorods obtained by solid-liquid reactions of $\mathrm{LaOCl}$ crystals. Chem. Mater. 22, 5411-5419 (2010)

37. Hou, $\mathrm{X}$. et al. Single-crystal $\mathrm{MoO}_{3}$ micrometer and millimeter belts prepared from discarded molybdenum disilicide heating elements. Sci. Rep. 8, 16771 (2018).

38. Wu, N. Q. et al. Shape-enhanced photocatalytic activity of single-crystalline anatase $\mathrm{TiO}_{2}$ (101) nanobelts. J. Am. Chem. Soc. 132, 6679-6685 (2010).

39. Boppella, R., Choi, C. H., Moon, J. \& Kim, D. H. Spatial charge separation on strongly coupled 2D-hybrid of $\mathrm{rGO} / \mathrm{La}_{2} \mathrm{Ti}_{2} \mathrm{O}_{7} / \mathrm{NiFe}-\mathrm{LDH}$ heterostructures for highly efficient noble metal free photocatalytic hydrogen generation. Appl. Catal. B: Environ. 239, 178-186 (2018).

40. Hu, S., Chi, B., Pu, J. \& Jian, L. Novel heterojunction photocatalysts based on lanthanum titanate nanosheets and indium oxide nanoparticles with enhanced photocatalytic hydrogen production activity. J. Mater. Chem. A 2 , 19260-19267 (2014)

41. Selloni, A. Crystal growth: anatase shows its reactive side. Nat. Mater. 7, 613-615 (2008).

42. Li, R. et al. Spatial separation of photogenerated electrons and holes among $\{010\}$ and $\{110\}$ crystal facets of $\mathrm{BiVO}_{4}$. Nat. Commun. 4, 1432 (2013). 